\title{
Hille-Nehari type oscillation and nonoscillation criteria for linear and half-linear differential equations
}

\author{
Jana Řezníčková1,* \\ ${ }^{1}$ Tomas Bata University in Zlin, Faculty of Applied Informatics, Department of Mathematics, Nad Stráněmi 4511, 76005 Zlín, Czech \\ Republic
}

\begin{abstract}
Differential equations attract considerable attention in many applications. In particular, it was found out that half-linear differential equations behave in many aspects very similar to that in linear case. The aim of this contribution is to investigate oscillatory properties of the second-order half-linear differential equation and to give oscillation and nonoscillation criteria for this type of equation. It is also considered the linear SturmLiouville equation which is the special case of the half-linear equation. Main ideas used in the proof of these criteria are given and Hille-Nehari type oscillation and nonoscillation criteria for the Sturm-Liouville equation are formulated. In the next part, Hille-Nehari type criteria for the half-linear differential equation are presented. Methods used in this investigation are based on the Riccati technique and the quadratic functional, that are very useful instruments in proving oscillation/nonoscillation both for linear and half-linear equation. Conclude that there are given further criteria which guarantee either oscillation or nonoscillation of linear and half-linear equation, respectively. These criteria can be used in the next research in improving some conditions given in theorems of this paper.
\end{abstract}

\section{Introduction}

In this paper we investigate oscillatory properties of the half-linear second-order differential equation of the form

$$
\left(r(t) \Phi\left(x^{\prime}\right)\right)^{\prime}+c(t) \Phi(x)=0
$$

where $\Phi(x):=|x|^{p-2} x, p>1, t \in I:=[T, \infty)$ and $r, c$ are real-valued continuous functions and $r(t)>0$.

Oscillation theory of (1) attracted considerable attention in the past years and it was shown that solutions of (1) behave in many aspects like those of the linear SturmLiouville differential equation

$$
\left(r(t) x^{\prime}\right)^{\prime}+c(t) x=0,
$$

which is the special case $p=2$ of (1).

The aim of this paper is to present some results of the investigation oscillatory properties of equation (1) in comparison with that one of (2).

Note that the term half-linear equations is motivated by the fact that the solution space of (1) has just one half of the properties which characterize linearity, namely homogeneity, but not additivity.

The paper is organized as follows. In Section 2 we present basic concepts and properties of solutions of (1) and (2). Section 3 is devoted to the investigation of properties of solutions of (1) and (2), in particular, we present oscillation criteria for (1) and (2). Section 4 gives some nonoscillation criteria for (1) and (2).

*Corresponding author:reznickova@utb.cz

\section{Preliminary results}

In this section we define basic concepts concerning the half-linear differential equation (1).

Definition 1 Two points $t_{1}, t_{2} \in \mathbb{R}$ are said to be conjugate relative to (1) if there exists a nontrivial solution $x$ of this equation such that $x\left(t_{1}\right)=x\left(t_{2}\right)=0$.

Definition 2 Equation (1) is said to be disconjugate on a closed interval $[a, b]$ if this interval contains no pair of points conjugate relative to (1) (i.e., every nontrivial solution has at most one zero in I). In the opposite case, (1) is said to be conjugate on I (i.e., there exists a nontrivial solution with at least two zeros in I).

Note that by a zero of a solution $x$ we mean such a $t_{0}$ that $x\left(t_{0}\right)=0$.

Theorem 1 Equation (1) is disconjugate on an interval $I=[a, b]$ if and only if every its nontrivial solution has at most one zero in $[a, b]$.

The following property of zeros of linearly independent solutions is one of the most characteristic properties which justifies the definition of oscillation/nonoscillation of the equation.It is known as the Sturmian separation theorem and reads as follows (see [5]).

Theorem 2 Let $t_{1}<t_{2}$ be two consecutive zeros of a nontrivial solution $x$ of (1). Then any other solution of this equation which is not proportional to $x$ has exactly one zero on $\left(t_{1}, t_{2}\right)$. 

form

Along with (1) consider another equation of the same

$$
\left(R(t) \Phi\left(y^{\prime}\right)\right)^{\prime}+C(t) \Phi(y)=0,
$$

where the functions $R$ and $C$ satisfy the same assumptions as $r$ and $c$ in (1). The next theorem is known as the Sturmian comparison theorem and reads as follows (see [5]).

Theorem 3 Let $t_{1}<t_{2}$ be two consecutive zeros of a nontrivial solution $x$ of (1) and suppose that

$$
C(t) \geq c(t), \quad r(t) \geq R(t)>0
$$

for $t \in\left[t_{1}, t_{2}\right]$. Then any solution of (3) has a zero in $\left(t_{1}, t_{2}\right)$ or it is a multiple of the solution $x$.

Definition 3 Equation of (1) is said to be nonoscillatory at $\infty$, if there exists $T_{0} \in \mathbb{R}$ such that (1) is disconjugate on $\left[T_{0}, T_{1}\right]$ for every $T_{1}>T_{0}$. In the opposite case, (1) is said to be oscillatory, i.e., if every nontrivial solution has infinitely many zeros tending to $\infty$.

The previous definition says that one solution of (1) is oscillatory if and only if any other solution of (1) is oscillatory. Oscillation of a nontrivial solution of (1) means the existence of zeros of this solution tending to $\infty$.

One of the most important tools in the investigation of the qualitative properties of solutions of (1) is the Riccati technique and variational principle.

Riccati substitution. Let $x$ be a solution of (2) such that $x(t) \neq 0$ in an interval $I$. Then $w(t)=\frac{r(t) x^{\prime}}{x}$ is a solution of the associated Riccati differential equation

$$
w^{\prime}+c(t)+\frac{w^{2}}{r(t)}=0 .
$$

Equation (2) is nonoscillatory if and only if there exists a solution $w$ of (5) defined for large $t$, and this is equivalent to the positivity of the quadratic functional

$$
\mathcal{F}(y ; T, \infty)=\int_{T}^{\infty}\left[r(t) y^{\prime 2}-c(t) y^{2}\right] d t>0
$$

over the class of continuously differentiable functions $y$ such that $y(T)=0$ and $y(t) \equiv 0$ on $\left[T_{1}, \infty\right)$ for some $T_{1}>T$.

Now we introduce the half-linear version of the Riccati type equation associated with equation (1). Let $x$ be a solution of (1) such that $x(t) \neq 0$ in an interval $I$. Then

$$
w(t)=\frac{r(t) \Phi\left(x^{\prime}(t)\right)}{\Phi(x(t))}
$$

is a solution of the Riccati type differential equation of the form

$$
w^{\prime}+c(t)+(p-1) r^{1-q}(t)|w|^{q}=0,
$$

where $q$ is the conjugate number of $p$, i.e., $\frac{1}{p}+\frac{1}{q}=1$.

Let us recall that the Riccati equation and the $p$-degree functional

$$
\mathcal{F}_{p}(y ; T, \infty)=\int_{T}^{\infty}\left(r(t)\left|y^{\prime}\right|^{p}-c(t)|y|^{p}\right) d t
$$

play the same role in the oscillation theory of (1) as (5) and (6) in the linear oscillation theory.

\section{Oscillation criteria}

In this section we present oscillation criteria for equations (2) and (1), respectively, see [9].

\subsection{Oscillation criteria for Sturm-Liouville equation}

Hille-Nehari type oscillation criteria are criteria formulated in terms of the asymptotic behavior of the functions

$$
\int^{t} r^{-1}(s) d s \int_{t}^{\infty} c(s) d s
$$

or

$$
\int_{t}^{\infty} r^{-1}(s) d s \int^{t} c(s) d s
$$

depending on the convergence/divergence of the integrals appearing in these formulas. Note that if both integrals $\int^{\infty} r^{-1}(t) d t=\infty, \int^{\infty} c(t) d t=\infty$, equation (2) is oscillatory by the Leighton-Wintner oscillation criterion, see [10]. For the case (9), the Hille-Nehari criterion reads as follows (see, e.g. [1, Chap. 2]).

Theorem 4 Suppose that

$$
\int^{\infty} r^{-1}(t) d t=\infty
$$

and the integral $\int^{\infty} c(t) d t$ is convergent. Equation (2) is oscillatory provided one of the following conditions holds:

(i) $c(t) \geq 0$ for large $t$ and

$$
\limsup _{t \rightarrow \infty}\left(\int^{t} r^{-1}(s) d s\right)\left(\int_{t}^{\infty} c(s) d s\right)>1,
$$

(ii)

$$
\liminf _{t \rightarrow \infty}\left(\int^{t} r^{-1}(s) d s\right)\left(\int_{t}^{\infty} c(s) d s\right)>\frac{1}{4} .
$$

PROOF. (i) We prove this statement using the variational principle, i.e., we find, for every $T \in \mathbb{R}$, a function $y \in W^{1,2}(T, \infty)$ with a compact support in $(T, \infty)$ such that the functional $\mathcal{F}(y ; T, \infty) \leq 0$. To this end, let $T \in \mathbb{R}$ be arbitrary, $T<t_{0}<t_{1}<t_{2}<t_{3}$, and let

$$
y(t)= \begin{cases}0 & T \leq t \leq t_{0}, \\ \int_{t_{0}}^{t} r^{-1}(s) d s\left(\int_{t_{0}}^{t_{1}} r^{-1}(s) d s\right)^{-1} & t_{0} \leq t \leq t_{1}, \\ 1 & t_{1} \leq t \leq t_{2}, \\ \int_{t}^{t_{3}} r^{-1}(s) d s\left(\int_{t_{2}}^{t_{3}} r^{-1}(s) d s\right)^{-1} & t_{2} \leq t \leq t_{3}, \\ 0 & t_{3} \leq t<\infty .\end{cases}
$$

By a direct computation, using the fact that $c(t) \geq 0$ for large $t$, we obtain

$$
\begin{gathered}
\mathcal{F}(y ; T, \infty) \leq\left(\int_{t_{0}}^{t_{1}} r^{-1}(s) d s\right)^{-1} \\
\left(\int_{t_{2}}^{t_{3}} r^{-1}(s) d s\right)^{-1}-\int_{t_{1}}^{t_{2}} c(s) d s
\end{gathered}
$$$$
=\left(\int_{t_{0}}^{t_{1}} r^{-1}(s) d s\right)^{-1}\left[1+\left(\int_{t_{0}}^{t_{1}} r^{-1}(s) d s\right)^{-1}\left(\int_{t_{2}}^{t_{3}} r^{-1}(s) d s\right)^{-1}\right.
$$ 


$$
\left.-\int_{t_{0}}^{t_{1}} r^{-1}(s) d s\left(\int_{t_{1}}^{t_{2}} c(s) d s\right)\right] .
$$

Now, by (12), there exists $\varepsilon>0$ such that the last term in the brackets is less than $-1-\varepsilon$ for $t_{1}$ and $t_{2}$ sufficiently large, and (11) implies that the middle term is less than $\varepsilon$ if $t_{3}$ is sufficiently large. Hence $\mathcal{F}(y ; T, \infty) \leq 0$ for $t_{1}, t_{2}, t_{3}$ chosen in this way.

(ii) This part of the proof is based on the Riccati technique. Suppose, by contradiction, that (2) is nonoscillatory and $w$ is a solution of the associated Riccati equation (5). Then, according to [6, Chap. XI], the solution $w$ can be expressed in the form

$$
w(t)=\int_{t}^{\infty} c(s) d s+\int_{t}^{\infty} \frac{w^{2}(s)}{r(s)} d s .
$$

Multiplying the last equation by $\int^{t} r^{-1}(s) d s$, we have

$$
\begin{aligned}
\left(\int^{t} r^{-1}(s) d s\right) w(t) & =\left(\int^{t} r^{-1}(s) d s\right)\left(\int_{t}^{\infty} c(s) d s\right) \\
& +\left(\int^{t} r^{-1}(s) d s\right)\left(\int_{t}^{\infty} \frac{w^{2}(s)}{r(s)} d s\right) .
\end{aligned}
$$

Suppose first that $\liminf _{t \rightarrow \infty}\left(\int^{t} r^{-1}(s) d s\right) w(t)=\lambda$ exists finite. Then, using (13), there exists $\varepsilon>0$ such that

$$
\begin{gathered}
\left(\int^{t} r^{-1}(s) d s\right) w(t) \geq \frac{1}{4}+\varepsilon+\left(\int^{t} r^{-1}(s) d s\right) \\
\times \int_{t}^{\infty}\left[\left(\int^{s} r^{-1}(\tau) d \tau\right)^{2} w^{2}(s) \cdot \frac{r^{-1}(s)}{\left(\int^{s} r^{-1}(\tau) d \tau\right)^{2}}\right] d s
\end{gathered}
$$

for large $t$, and hence, letting $t \rightarrow \infty$ in the last inequality,

$$
\lambda \geq \frac{1}{4}+\varepsilon+\lambda^{2}
$$

i.e., $-\left(\lambda-\frac{1}{2}\right)^{2} \geq \varepsilon$, which is a contradiction.

If

$$
\liminf _{t \rightarrow \infty}\left(\int^{t} r^{-1}(s) d s\right) w(t)=\infty,
$$

denote

$$
m(t)=\inf _{t \leq s}\left(\int_{T}^{s} r^{-1}(\tau) d \tau\right) w(s)
$$

Then $m$ is nondecreasing and using (13) there exists $\varepsilon>0$ such that

$$
\begin{gathered}
\left(\int^{t} r^{-1}(s) d s\right) w(t) \geq \frac{1}{4}+\varepsilon+\left(\int^{t} r^{-1}(s) d s\right) \\
\times \int_{t}^{\infty} m^{2}(t) \cdot \frac{r^{-1}(s)}{\left(\int^{s} r^{-1}(\tau) d \tau\right)^{2}} d s,
\end{gathered}
$$

which means

$$
\left(\int^{t} r^{-1}(s) d s\right) w(t) \geq \frac{1}{4}+\varepsilon+m^{2}(t) .
$$

Since $m$ is nondecreasing, we have for $s>t$

$$
m(s) \geq \frac{1}{4}+\varepsilon+m^{2}(t)
$$

thus,

$$
m(t) \geq \frac{1}{4}+\varepsilon+m^{2}(t)
$$

which is a contradiction with (14).

\subsection{Oscillation criteria for half-linear equation}

Now, we turn out attention to Hille-Nehari type oscillation criteria for the half-linear differential equation (1).

A direct modification of the proof of Theorem 4 shows that the criteria given in that theorem can be extended to (1) as follows, see [5, Sec 3.1.1].

Theorem 5 Suppose that $\int^{\infty} r^{1-q}(t) d t=\infty$ and the integral $\int^{\infty} c(t) d t$ is convergent. Equation (1) is oscillatory provided one of the following conditions holds:

(i) $c(t) \geq 0$ for large $t$ and

$$
\limsup _{t \rightarrow \infty}\left(\int^{t} r^{1-q}(s) d s\right)^{p-1}\left(\int_{t}^{\infty} c(s) d s\right)>1,
$$

(ii)

$$
\liminf _{t \rightarrow \infty}\left(\int^{t} r^{1-q}(s) d s\right)^{p-1}\left(\int_{t}^{\infty} c(s) d s\right)>\frac{1}{p}\left(\frac{p-1}{p}\right)^{p-1} .
$$

Note that in the modification of the part (ii) of the proof of Theorem 5, one needs to use the fact that

$$
|\lambda|^{q}-\lambda=\frac{1}{p}\left(\frac{p-1}{p}\right)^{p-1} \geq 0 \text { for every } t \in \mathbb{R} .
$$

\section{Nonoscillation criteria}

In this part we present Hille-Nehari type nonoscillation criteria for equation (1). We also present a brief survey of linear version of Hille-Nehari criteria.

\subsection{Nonoscillation criterion for Sturm-Liouville equation}

In this subsection we formulate a nonoscillation criterion for (2) with $\int^{\infty} r^{-1}(t) d t=\infty$. (A similar nonoscillation criterion can be formulated also in the case when $\int^{\infty} r^{-1}(t) d t<\infty$, see [7]).

Theorem 6 Suppose that $\int^{\infty} r^{-1}(t) d t=\infty$ and $\int^{\infty} c(t) d t<\infty$. If

$$
\begin{array}{r}
\limsup _{t \rightarrow \infty}\left(\int^{t} r^{-1}(s) d s\right)\left(\int_{t}^{\infty} c(s) d s\right)<\frac{1}{4}, \\
\liminf _{t \rightarrow \infty}\left(\int^{t} r^{-1}(s) d s\right)\left(\int_{t}^{\infty} c(s) d s\right)>-\frac{3}{4},
\end{array}
$$

then (2) is nonoscillatory.

PROOF. It is well known from the linear Sturmian theory that equation (2) is nonoscillatory provided there exists a differentiable function $u$ which satisfies the Riccati-type inequality

$$
u^{\prime}+c(t)+\frac{u^{2}}{r(t)} \leq 0
$$


for large $t$ (see [6, Chap. XI]).

We will show that the function

$$
u(t)=\frac{1}{4 \int^{t} r^{-1}(s) d s}+\int_{t}^{\infty} c(s) d s
$$

satisfies (19) for large $t$. To this end, denote

$$
G(t):=\int^{t} r^{-1}(s) d s, \quad C(t):=\int_{t}^{\infty} c(s) d s
$$

thus,

$$
u(t)=\frac{1}{4 G(t)}+C(t)
$$

Then we have

$$
\begin{aligned}
u^{\prime}+c(t)+\frac{u^{2}}{r(t)} & =-\frac{r^{-1}(t)}{4 G t(t}-C(t)+C(t) \\
& +\frac{(1+4 G(t) C(t))^{2}}{16 G^{2}(t) r(t)} \\
& =\frac{r^{-1}(t)}{4 G^{2}(t)}\left[-1+\frac{(1+4 G(t) C(t))^{2}}{4}\right] .
\end{aligned}
$$

Now, since (17) and (18) hold, there exists $\delta>0$ such that

$$
\begin{gathered}
-\frac{3+\delta}{4}<G(t) C(t)<\frac{1+\delta}{4} \Longleftrightarrow \\
|1+4 G(t) C(t)|<2-\delta \Longleftrightarrow \frac{(1+4 G(t) C(t))^{2}}{4}<1 .
\end{gathered}
$$

Consequently, we have

$$
u^{\prime}+c(t)+\frac{u^{2}}{r(t)} \leq 0
$$

thus, (2) is nonoscillatory.

\subsection{Nonoscillation criteria for half-linear equation}

Before formulating Hille-Nehari type nonoscillation criteria for (1), let us recall that the basic facts of the half-linear oscillation theory can be found in [5].

Now, we present the main results which are formulated and proved in [3],[8].

Theorem 7 Suppose that $\int^{\infty} r^{-1}(t) d t=\infty$ and $\int^{\infty} c(t) d t<\infty$. If

$$
\limsup _{t \rightarrow \infty}\left(\int^{t} r^{1-q}(s) d s\right)^{p-1}\left(\int_{t}^{\infty} c(s) d s\right)<\frac{1}{p}\left(\frac{p-1}{p}\right)^{p-1},
$$

and

$$
\begin{gathered}
\liminf _{t \rightarrow \infty}\left(\int^{t} r^{1-q}(s) d s\right)^{p-1}\left(\int_{t}^{\infty} c(s) d s\right) \\
>-\frac{2 p-1}{p}\left(\frac{p-1}{p}\right)^{p-1},
\end{gathered}
$$

then (1) is nonoscillatory.
The next theorem deals with the case $\int^{\infty} r^{1-q}(t) d t<\infty$. Theorem 8 Suppose that $\int^{\infty} r^{-1}(t) d t<\infty$. If

$$
\underset{t \rightarrow \infty}{\limsup }\left(\int_{t}^{\infty} r^{1-q}(s) d s\right)^{p-1}\left(\int^{t} c(s) d s\right)<\frac{1}{p}\left(\frac{p-1}{p}\right)^{p-1},
$$

and

$$
\begin{gathered}
\liminf _{t \rightarrow \infty}\left(\int_{t}^{\infty} r^{1-q}(s) d s\right)^{p-1}\left(\int_{t}^{\infty} c(s) d s\right) \\
>-\frac{2 p-1}{p}\left(\frac{p-1}{p}\right)^{p-1}
\end{gathered}
$$

then (1) is nonoscillatory.

The proof of these statements follows the idea introduced in the proof of Theorem 6 . We put

$$
w=\left(\frac{p-1}{p}\right)^{p}\left(\int^{t} r^{1-q}(s) d s\right)^{1-p}+\int_{t}^{\infty} c(s) d s
$$

in the proof of Theorem 7 and

$$
w=-\left(\frac{p-1}{p}\right)^{p}\left(\int_{t}^{\infty} r^{1-q}(s) d s\right)^{1-p}+\int^{t} c(s) d s
$$

in the proof of Theorem 8 . By a direct computation similar to that in the proof of Theorem 6 one can show that

$$
w^{\prime}+c(t)+(p-1) r^{1-q}(t)|w|^{q} \leq 0
$$

and this implies, by [5, Theorem 2.2.1], that (1) is nonoscillatory.

\section{References}

[1] R. P. Agarval, S. R. Grace, D. O'Regan, Oscillation theory for Second Order Linear, Half-Linear, Superlinear and Sublinear Dynamic Equations (Kluwer Academic Publishers, Dordrecht/Boston/London, 2002).

[2] O. Došlý, 1, 161-357 (2004).

[3] O. Došlý, 50, 657-671 (2000).

[4] O. Došlý, A. Lomtatidze, 36, 203-219 (2006).

[5] O. Došlý, P. Řehák, Half-Linear Differential Equations (North Holland Mathematics Studies 202, Elsevier, Amsterdam, 2005).

[6] P. Hartman, Ordinary Differential Equations (John Wiley, New York, 1964).

[7] E. Hille, 64, 234-252 (1948).

[8] J. Řezníčková, 15, 55-62 (2006).

[9] J. Řezníčková, 16, 139-147 (2007).

[10] C. A. Swanson, Comparison and Oscillation Theory of Linear Differential Equations, Acad. Press, New York-London (1968). 